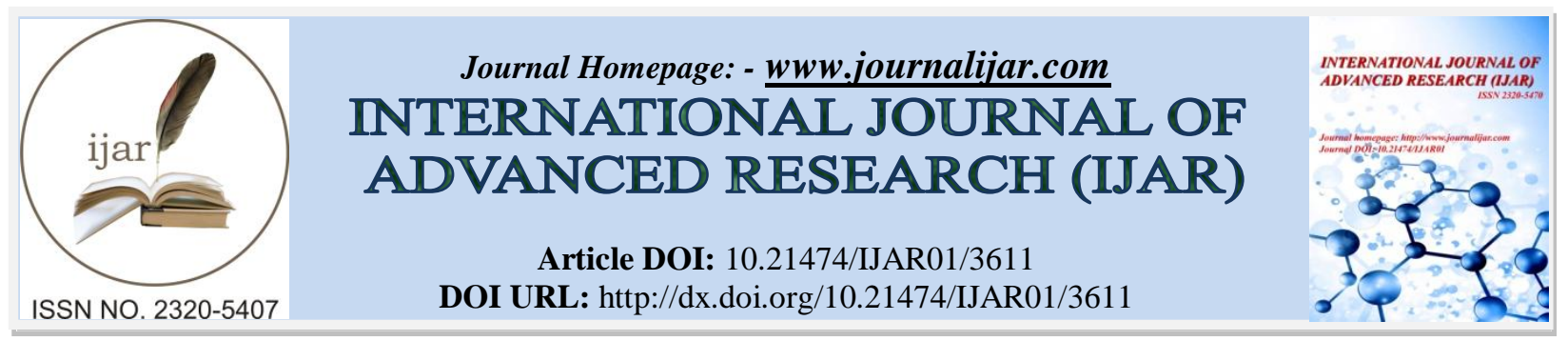

RESEARCH ARTICLE

\title{
EVALUATION OF GRADIENT GEOMETRY FOR MULTIMODAL MEDICAL IMAGE FUSION
}

Jael Edith. N, Nithya. B and Palani Thanaraj. K.

Department of Electronics and Instrumentation Engineering, St.Joseph's College of Engineering, Chennai-600119, Tamilnadu, India.

\section{Manuscript Info}

Manuscript History

Received: 07 January 2017

Final Accepted: 10 February 2017

Published: March 2017

Key words:-

Medical image fusion, geometry, SSIM, PSNR .

\section{Abstract}

Fusion of medical images such as MRI and PET by gradient selection is attempted in this paper. The efficiency of the fusion process depends on the selection of the appropriate image geometry. This work analyses different gradient geometries for medical image fusion. From the experimental analysis, it is found that the images fused by the Southwell geometry produced better results when compared to Fried geometry and Hudgin geometry.

Copy Right, IJAR, 2017,. All rights reserved.

\section{Introduction:-}

Image fusion integrates information from multiple individual images into a single image so that the obtained image contains more details of the source images. In static image fusion, it is necessary that the input images are aligned and there exists coincidence in terms of depth or field of view of the imaged scenes. Image registration is done to make the images suitable for static image fusion [1]. Image fusion algorithm can be classified into pixel-based and regional-based algorithm [2].In pixel-based methods, each pixel of the input images are combined using various rules such as averaging, finding maximum to form corresponding pixels in the fused image [3]. In region-based techniques, wavelet transforms are used to represent the input images in a multi-resolution framework, and then each region of the image is applied a different fusion rules that are used to desegregate the information into a more comprehensive image [4].

Medical image fusion [5] aids in the clinical diagnosis and treatment by providing useful information especially for lesion location, making treatment and pathological study. MRI image gives information about anatomical structure of soft tissues such as brain, organs and blood vessels [6,7], while PET image suffers from low resolution but provides physiological information [8].Hence fusion of these two images offers the advantage of better diagnosis of diseases [9] particularly of the cancer tumours.

Socolinsky and Wolff proposed an image fusion approach in which the information from a multi-spectral image dataset is integrated to produce a one band display of the image. They generalize image gradients are those which closely related to image contrast [10], by defining it for multi-spectral images in terms of differential geometry. The desirable gradient field is reconstructed using the contrast information, to produce the fused image. Later, Wang $e t$ al fused the images in gradient domain using weights dependent on local variations in intensity of the input images [11]. At each pixel position, they construct an importance-weighted contrast matrix.

The algorithm proposed by Sujoy Paul et al combines gradient fusion as well as pixel-based fusion [12]. It works for the fusion of colour or grayscale image for multi-focal, multi resolution [13] and multi-exposure [14] images. In the 
case of colour images, the luminance and chrominance of the images are fused in a different manner. By doing so the computational time for running the algorithm is greatly reduced. Also, due to the fact that the luminance (Y) channel represents the image brightness and contrast information and it is in this channel where variations and details are most visible, since the human visual system is more sensitive to luminance $(\mathrm{Y})$. The chrominance $(\mathrm{Cb}, \mathrm{Cr})$ [15] channels contain only colour information, to which the human visual system is less sensitive. Comparison between Hudgin, Fried, Southwell geometries proved the advantage of each for noise reduction. Roopashree et al reported that the fried geometry produced worst performance, as its consistency is poor [16].

In this paper, the fusion of the medical image using the gradient selection is proposed. The quality of the image is analyzed by using different geometry. The performance assessment test such as Structural Similarity Index (SSIM), and Peak Signal to Noise Ratio (PSNR)are used for testing the accuracy of the fusion process.

\section{Materials and Methods:-}

The static image fusion requires input multi-modal images of same size and in the same depth of view. Hence input image must be registered before processing [17]. Here, pre-registered MRI and PET images are taken for further processing.

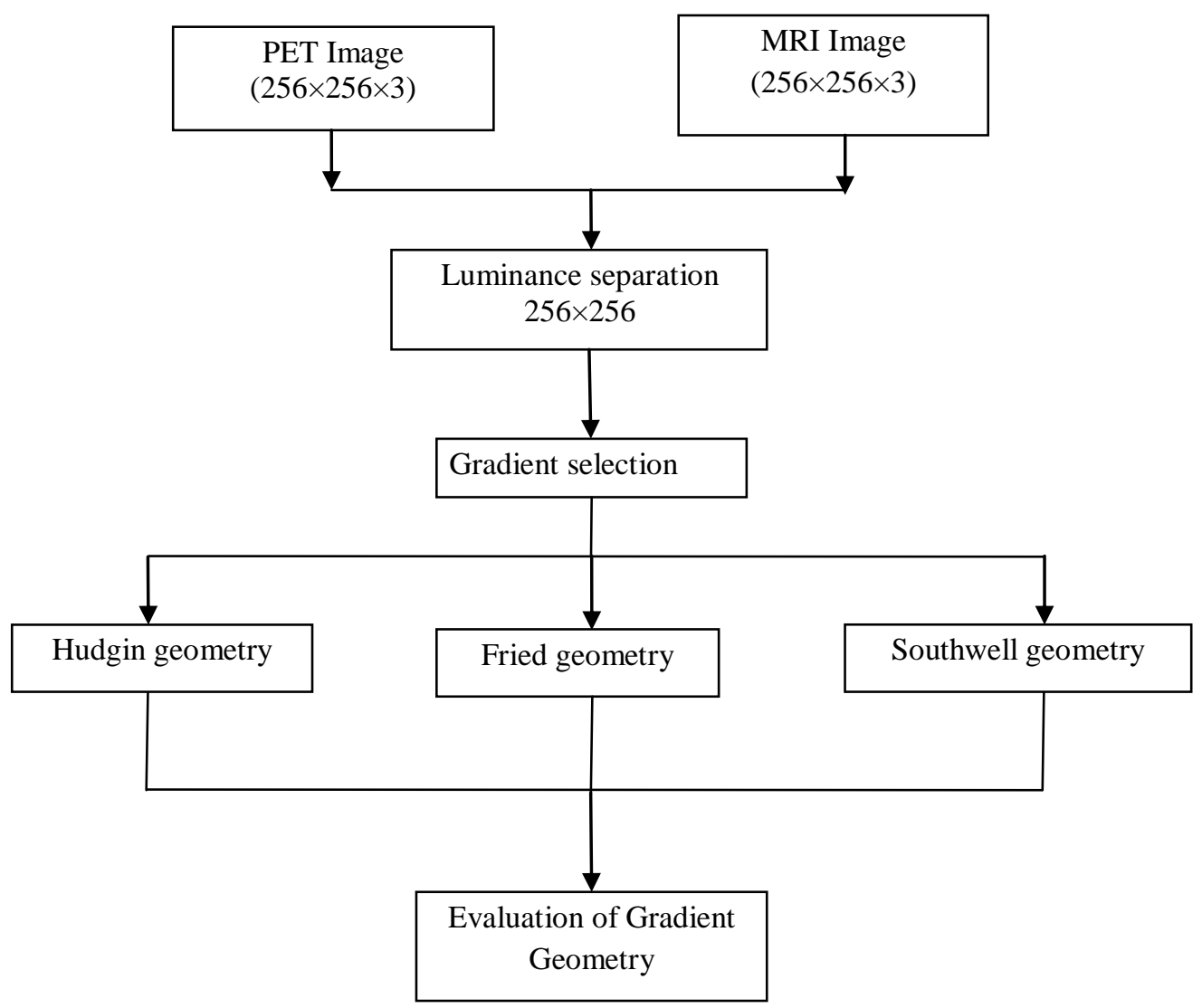

Fig. 1:- Steps involved in the evaluation of gradient geometry for medical image fusion

The input images are taken from www.med.harvard.edu .The Fig. 1.explains the gradient selection process. Initially, luminance channel is separated from each of the images. From that the gradients are selected using various geometries such as Hudgin geometry, Fried geometry, Southwell geometry. After fusion of images using any reconstruction [18] algorithm, the parameters PSNR and SSIM are calculated for each fused image. Finally, the performance of the fused image is compared and analyzed.

Fusion technique proposed by Sujoy paul et al for colour image the luminance part is separated from the chrominance part (RGB to $\mathrm{YCbCr}$ ) as different fusion technique is followed for each. Gradient fusion is carried out 
for luminance channel. This involves taking the gradients with the maximal magnitude at each pixel position will lead to an image which has much more detail than any other image in the stack. Luminance channel for $\mathrm{N}$ input images are stored in a stack as $I^{\prime}=\left\{I_{1}, I_{2}, \ldots I_{N}\right\}$ where $N>2$. The gradient value for each image is found for each pixel position using Hudgin geometry. Those values are taken mean with the neighbouring pixels and are made equivalent for the gradient for the respective pixels which is the Southwell geometry explained later in this paper.

The gradient of the luminance channel of an image may be $\Phi_{n}^{x}(x, y)$ and $\Phi_{n}^{y}(x, y)$ where $\Phi_{n}^{x}$ and $\Phi_{n}^{y}$ are the gradient components along the $\mathrm{x}$ and $\mathrm{y}$ directions respectively.

The magnitude of the gradient may be

$$
\left.\left.H_{n}(x, y)=\sqrt{\left(\left(\Phi_{n}^{x}\right.\right.}(x, y)\right)^{2}+\left(\Phi_{n}^{y}(x, y)\right)^{2}\right)
$$

The magnitude of gradient at all the pixel positions of each image is compared with that of the other images and the maximum value of that is stored as

$$
P(x, y)=\max _{1 \leq n \leq N} H_{n}(x, y)
$$

The fused luminance gradient is

$$
\begin{aligned}
& \Phi^{x}(x, y)=\Phi_{p(x, y)}^{x}(x, y) \\
& \Phi^{y}(x, y)=\Phi_{p(x, y)}^{y}(x, y)
\end{aligned}
$$

where $\Phi^{x}(x, y)$ and $\Phi^{y}(x, y)$ are the values of gradient components in the $\mathrm{x}$ and $\mathrm{y}$ gradient components of the image with index $P(x, y)$ at pixel location $(x, y)$ and the fused luminance gradient is $\Phi=\left[\Phi_{x} \Phi_{y}\right]^{T}$. The relationship between the fused gradient $(\Phi)$ and the luminance channel $(I)$ is $\nabla I=\Phi$, where $\nabla=[d / d x, d / d y]^{T}$.

As the fused gradient is a combination of multiple luminance channel, there are possibilities for high peaks in the adjacent pixels. Hence it needs to be optimized using poisson solver which satisfies the zero curl condition to remove artifacts. The reconstruction of the luminance channel from the gradient data is done with a wavelet based algorithm proposed by Haar-Hampton et al followed by image enhancement using gamma correction. The fusion procedure for grayscale images is the same as followed for the luminance channel.

For chrominance part, the individual chrominance channels $\left(C_{b}, C_{r}\right)$ are taken and the fusion is carried out by taking the weighted sum, with the weights depending on the channel intensities of input chrominance channels. Their values will range from 16 to 240 . For grayscale images this process does not apply as they have values of both $C_{b}$ and $C_{r}$ equal to 128 . The weights must be selected for pixel positions such that how far the chrominance value is from 128. Computation is less intensive than gradient fusion.

\section{Concept of Geometries:-}

Local gradients are measured at discrete locations of the wavefront. Depending on the position at which phase differences are estimated with reference to the location of slope measurements, there exist three main classifications in wavefront sampling namely Fried, Hudgin and Southwell (or Shack Hartmann) geometries. The illustration of relationship between the slope and phase measurements for the above geometries are shown in fig(1). 


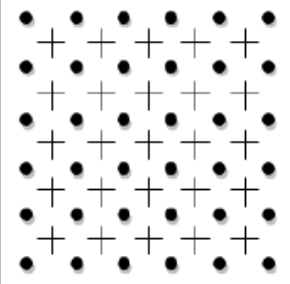

(a)

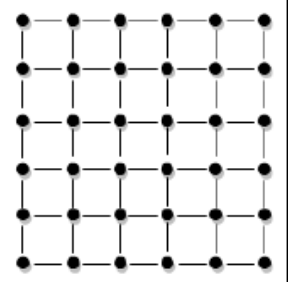

(b)

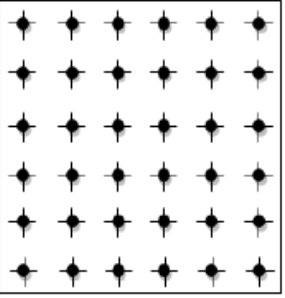

(c)

Fig.2:- Sampling Geometry (a) Fried (b) Hudgin (c) Southwell

The horizontal and vertical lines represent positions of slope measurements in $\mathrm{x}$ and $\mathrm{y}$ directions respectively and dots represent positions of phase estimation. The difference between the geometries are shown in Fig.2. In Fried and Southwell configurations both $\mathrm{x}$ and $\mathrm{y}$ slope measurements are made at the same point. In the Southwell (Shack Hartmann) configuration, slope measurement points coincide with the phase evaluation points whereas in Fried configuration, phase evaluation grid is displaced by half the sensor pitch with respect to slope measurement grid. In Hudgin geometry, $\mathrm{x}$ and $\mathrm{y}$ slope measurement points are displaced from one another and phase is evaluated at the edges of the slopes [19]. The slope to phase relation for different configurations are as follows

For Fried geometry,

$$
\begin{aligned}
& \Phi_{n}^{x}(x, y)=\frac{\left[\frac{\left.\Phi_{(x+1, y)}+\Phi_{(x+1, y+1)}-\frac{\Phi_{(x, y)}+\Phi_{(x, y+1)}}{2}\right]}{h}\right.}{h} \\
& \Phi_{n}^{y}(x, y)=\frac{\left[\frac{\Phi_{(x, y+1)}+\Phi_{(x+1, y+1)}}{2}-\frac{\Phi_{(x, y)}+\Phi_{(x+1, y)}}{2}\right.}{h}
\end{aligned}
$$

Where $x=1,2, \ldots \ldots N-1$ and $y=1,2, \ldots . . N-1$.

For Hudgin geometry,

$$
\begin{aligned}
& \Phi_{n}^{x}(x, y)=\frac{\Phi_{(x+1, y)}+\Phi_{(x, y)}}{h} \\
& \Phi_{n}^{y}(x, y)=\frac{\Phi_{(x, y+1)}+\Phi_{(x, y)}}{h}
\end{aligned}
$$

Where $x=1,2, \ldots . N-1$ and $y=1,2, \ldots \ldots N$.

For Southwell geometry,

$$
\begin{aligned}
& \frac{\Phi_{n}^{x}(x+1, y)+\Phi_{n}^{x}(x, y)}{2}=\frac{\Phi_{(x+1, y)}+\Phi_{(x, y)}}{h} \\
& \frac{\Phi_{n}^{y}(x, y+1)+\Phi_{n}^{y}(x, y)}{2}=\frac{\Phi_{(x, y+1)}+\Phi_{(x, y)}}{h}
\end{aligned}
$$

Where $x=1,2, \ldots \ldots N$ and $y=1,2, \ldots . N-1$.

Any of the above geometries can be applied to find the gradient values from the input images each producing different quality of fused image when the gradient data is used for reconstruction.

\section{Image Quality Assessment Tests:-} (i)PSNR:

The Peak Signal to Noise Ratio(PSNR) can be computed by calculating MSE(Mean Square Error). 
It can be calculated by squaring the intensities of original input images and the final output image[20]. Then the average is taken. It is given by

$$
M S E=\left(\frac{1}{N M}\right) \sum_{m=0}^{M-1} \sum_{n=0}^{N-1} e(m, n)^{2}
$$

Where $e(m, n)$ is the error difference between original and distorted images. PSNR is obtained as the pixel difference between the reconstructed and the original image. It is given by

$$
P S N R=10 \log \frac{S^{2}}{M S E}
$$

where $\mathrm{S}=255$ for images having 8 -bit.

\section{(ii)SSIM:}

The Structural SIMilarity Index (SSIM) is a method for measuring similarity between two images. The SSIM index can be viewed as a quality measure in which the fused image is compared with the image that is considered as perfect quality [21]. Its formula is given as

$$
\operatorname{SSIM}(x, y)=\frac{\left(2 \mu_{x} \mu_{y}+C_{1}\right)\left(2 \sigma_{x y}+C_{2}\right)}{\left(\mu_{x}^{2}+\mu_{y}^{2}+C_{1}\right)\left(\sigma_{x}^{2}+\sigma_{y}^{2}+C_{2}\right)}
$$

\section{Results and Discussion:-}

The fusion of medical images (MRI and PET) based on gradient approach is presented in this paper. The gradient approach is done only to Luminance channel. The input gradients are taken using three different images such as Hudgin geometry, Fried geometry, Southwell geometry. Among these three methods Southwell geometry provides the best PSNR and SSIM values. The comparison among the geometries for five different cases are given below.

In Table 1, the normal brain images from MRI and PET are taken and fused using three different geometries. From the fused image of Fried geometry it is shown that the local features of the input images are not obtained clearly. The fused images of Hudgin geometry and Southwell geometry seem to be similar, but the parameters PSNR and SSIM is high for Southwell geometry compared to Hudgin geometry.

In Table 2, the PSNR value of fused image of Fried geometry is very much lower compared to Southwell geometry. While considering Fried geometry, there are more undesirable artifacts and so it is better to use Southwell geometry. SSIM of Southwell geometry is also greater to some extent when compared with other geometries. It can be seen that the tumour is more clearly visible in Southwell geometry image.

In Table 3, the twists and turns of the brain tissues are clearly visible in the fused image of Southwell geometry. But they are not visible in Fried geometry. Southwell geometry is having PSNR and SSIM value very much better than Fried geometry. SSIM value of Southwell geometry is nearly 100 times better than Fried geometry.

In Table 4, Carcinoma is visible more obvious in the fused image of Southwell geometry. Noise ratio and similarity index are higher when compared to other two geometries which may result in better diagnosis of the disease.

In Table 5, Similarity index is very poor in Fried geometry. The features of the original images are not clearly visible. It will be difficult for doctors to diagnose. 
Table 1:- Fusion of MRI/PET images of Normal Brain

\begin{tabular}{|c|c|c|c|c|c|}
\hline CASE & MRI & PET & $\begin{array}{l}\text { HUDGIN } \\
\text { GEOMETRY }\end{array}$ & $\begin{array}{l}\text { FRIED } \\
\text { GEOMETRY }\end{array}$ & $\begin{array}{l}\text { SOUTHWELL } \\
\text { GEOMETRY }\end{array}$ \\
\hline $\begin{array}{l}\text { NORM } \\
\text { L BRA }\end{array}$ & & & & & \\
\hline PSNR & & & 18.3559 & 12.2044 & 18.9041 \\
\hline SSIM & & & 0.4686 & 0.0069 & 0.4872 \\
\hline
\end{tabular}

Table 2:- Fusion of MRI/PET images of Brain Tumour

\begin{tabular}{|c|c|c|c|c|c|}
\hline CASE & MRI & PET & $\begin{array}{l}\text { HUDGIN } \\
\text { GEOMETRY }\end{array}$ & $\begin{array}{l}\text { FRIED } \\
\text { GEOMETRY }\end{array}$ & $\begin{array}{l}\text { SOUTHWELL } \\
\text { GEOMETRY }\end{array}$ \\
\hline $\begin{array}{l}\text { BRAIN } \\
\text { TUMO } \\
\text { R }\end{array}$ & & & & & \\
\hline PSNR & & & 18.024 & 14.0082 & 22.5549 \\
\hline SSIM & & & 0.2320 & -0.0324 & 0.2775 \\
\hline
\end{tabular}

Table 3:- Fusion of MRI/PET images of Motor Neuron disease

\begin{tabular}{|c|c|c|c|c|c|}
\hline CASE & MRI & PET & $\begin{array}{l}\text { HUDGIN } \\
\text { GEOMETRY }\end{array}$ & $\begin{array}{l}\text { FRIED } \\
\text { GEOMETRY }\end{array}$ & $\begin{array}{l}\text { SOUTHWELL } \\
\text { GEOMETRY }\end{array}$ \\
\hline $\begin{array}{l}\text { MOTO } \\
\text { NEUR( } \\
\text { DISEA }\end{array}$ & & & & & \\
\hline PSNR & & & 21.6072 & 14.3681 & 21.9384 \\
\hline SSIM & & & 0.4041 & -0.0421 & 0.4213 \\
\hline
\end{tabular}

Table 4:- Fusion of MRI /PET images of Metastatic Bronchogenic Carcinoma

\begin{tabular}{|l|l|l|l|l|l|}
\hline CASE & MRI & PET & $\begin{array}{l}\text { HUDGIN } \\
\text { GEOMETRY }\end{array}$ & $\begin{array}{l}\text { FRIED } \\
\text { GEOMETRY }\end{array}$ & $\begin{array}{l}\text { SOUTHWELL } \\
\text { GEOMETRY }\end{array}$ \\
\hline META & STATIC \\
BRONCHO & GENIC \\
CARCINOMA & & &
\end{tabular}


Table 5:- Fusion of MRI/PET images of Hypertensive Encephalopathy

\begin{tabular}{|c|c|c|c|c|c|}
\hline CASE & MRI & PET & $\begin{array}{l}\text { HUDGIN } \\
\text { GEOMETRY }\end{array}$ & $\begin{array}{l}\text { FRIED } \\
\text { GEOMETRY }\end{array}$ & $\begin{array}{l}\text { SOUTHWELL } \\
\text { GEOMETRY }\end{array}$ \\
\hline $\begin{array}{l}\text { HYPEI } \\
\text { ENCEI } \\
\text { HY }\end{array}$ & & & & & \\
\hline PSNR & & & 19.7199 & 13.2178 & 20.2520 \\
\hline SSIM & & & 0.3759 & -0.0324 & 0.2775 \\
\hline
\end{tabular}

\section{Conclusion:-}

This work has demonstrated the fusion of MRI and PET images using Gradient Selection method. The luminance channels of MRI and PET are taken and the gradients are selected using three geometries namely Hudgin geometry, Fried geometry, and Southwell geometry. The performances of the three geometries are analyzed using the parameters PSNR and SSIM. Based on the comparative analysis, it is found that the Southwell geometry provides better results for fusion of MRI and PET images.

\section{References:-}

1. Sombir Singh Bisht et al "Int. Journal of Engineering Research and Applications", Vol. 4, Issue 4( Version 7), April 2014, pp.30-35.

2. Priya D. Vora, Ms. Neeta Chudasama, "Different Image Fusion Techniques and Parameters: A Review", International Journal of Computer Science and Information Technologies, Vol. 6 (1), 2015, 889-892

3. B. Yang and $\mathrm{S}$. Li, "Pixel-level image fusion with simultaneous orthogonal matching pursuit," Information Fusion, vol. 13, no. 1, pp. 10-19, 2012.

4. J. Yang and R. S. Blum, A region-based image fusion method using the expectation maximization algorithm, Annual Conf. Information Sciences and Systems (Princeton, NJ, 2006), pp. 468-473.

5. "Image Fusion of PET and CT Images based on Wavelet Transform" by Jitendra Gangwar M.Tech Scholar ECE Department SHIATS-DU Allahabad, U.P., India ,Anil Kumar Assistant Professor ECE Department SHIATS-DU Allahabad, U.P., India, A.K.Jaiswal, Professor, H.O.D. ECE Department SHIATS-DU Allahabad, U.P., India.

6. M.Ramamoorthy, K.Anees Barvin, "Medical Image Fusion Using Gabor And Gradient Measurement", International Journal of Innovative Research in Science, Engineering and Technology, Volume 3, Special Issue 3, March 2014 ISSN (Print) : $2347-6710$.

7. M. Ashwin Kumaar and Palani Thanaraj, "Feature Extraction of Arterio-Venous Malformation Images using Grey Level Co-Occurrence Matrix", Indian Journal of Science and Technology, Vol 8(35), ISSN (Print) : 09746846, DOI:10.17485/ijst/2015/v8i35/83387, December 2015.

8. Umer Javed, Muhammad Mohsin Riaz, Abdul Ghafoor, Syed Sohaib Ali, and Tanveer Ahmed Cheema, "MRI and PET Image Fusion Using Fuzzy Logic and Image Local Features", Hindawi Publishing Corporation the Scientific World Journal Volume 2014, Article ID 708075, 8 pages, 19 January 2014.

9. Hossein Jadvar, Patrick M.Colletti, Division of Nuclear Medicine of USC, University of southern California, "Competitive advantage of PET/MRI", Published by Elsevier Inc., 2013.

10. Socolinsky, D.A., Wolff, L.B., "Multispectral Image Visualization through First-order fusion”, IEEE Trans. Im. Proc. 11, 923-931 (2002).

11. C. Wang, Q. Yang, X. Tang and Z. Ye, Salience preserving image fusion with dynamic range compression, IEEE Int. Conf. Image Process. (Atlanta, GA, 2006), pp. 989-992.

12. Sujoy Paul, Ioana S. Sevcenco and Panajotis Agathoklis, "Multi-Exposure and Multi-Focus Image Fusion in gradient domain", Journal of Circuits, Systems, and Computers Vol. 25, No. 10 (2016) 1650123 (18 pages)

13. Valdimir S. Petrovic', and Costas S. Xydeas, "Gradient-Based Multiresolution Image Fusion", IEEE Transactions on image processing, VOL. 13, NO. 2, February 2004.

14. K. Ma and Z. Wang, Multi-exposure image fusion: A patch-wise approach, IEEE Int.Conf. Image Processing (Quebec City, QC, 2015), pp. 1717-1721.

15. Douglas A. Kerr, P.E., "Chromaticity and Chrominance in Color Definition”, Issue 3, August 9, 2003 
16. M.B.Roopashree, Akondi Vyas, B.R Prasad, "Performance analysis of Fourier and Vector Matrix Multiply methods for phase reconstruction from slope measurements", ICOP 2009-International Conference on Optics and Photonics Chandigarh, India, 30 Oct.-1 Nov.2009.

17. Sombir Singh Bisht et al Int. Journal of Engineering Research and Applications www.ijera.com ISSN : 22489622, Vol. 4, Issue 4( Version 7), April 2014, pp.30-35.

18. I.S.Sevcenco, P.J.Hampton, P.Agathoklis, "A wavelet based method for image reconstruction from gradient data with applications", Multidimensional system and signal processing

19. Shane C.Moran, United States Naval Academy, "Comparison of a noise variance weighted complex exponential reconstructor with traditional reconstruction in the presence of deep turbulence", June 2013, Form approved OMB No.0704-0188.

20. Yusra A. Y. Al-Najjar, Dr. Der Chen Soong, "Comparison of Image Quality Assessment: PSNR, HVS, SSIM, UIQI", International Journal of Scientific \& Engineering Research, Volume 3, Issue 8, August-2012, ISSN 2229-5518.

21. Z. Wang, A.C. Bovik, H. R. Sheikh and E. P. Simoncelli, "Image quality assessment: From error visibility to structural similarity", Image Processing IEEE Transactions on, vol. 13, no. 4, (2004), pp. 600-612. 